\title{
Unilateral Idiopathic Dilated Episcleral Vein with Secondary Open Angle Glaucoma (Radius-Maumenee Syndrome) in an African - A case report and literature review
}

\author{
TO Otulana, OO Onabolu, VO Fafiolu
}

Department of Ophthalmology, Faculty of Clinical Sciences, Obafemi Awolowo College of Health Sciences, Olabisi Onabanjo University, Sagamu

\begin{abstract}
A 69-year-old woman was seen in the eye clinic of the Olabisi Onabanjo University Teaching Hospital [OOUTH], Sagamu, south-west Nigeria, with irritation in the right eye due to a vascular nasal pterygium, but asymptomatic in her left eye. Examination revealed dilated and tortuous conjunctival and episcleral vessels in her left eye with no apparent cause. She later developed secondary glaucoma in that eye. A diagnosis of left idiopathic dilated episcleral vein with secondary open angle glaucoma [Radius Maumenee syndrome] was made. She was treated both medically and surgically. This rare case in an African woman is presented in order to highlight the possibility of secondary vascular glaucoma in a patient with chronic redness of the eye without symptoms of discomfort or allergy.
\end{abstract}

Key words: idiopathic, dilated episcleral veins, secondary glaucoma, Nigeria

\section{INTRODUCTION}

Idiopathic dilated episcleral vein (IDEV) is an uncommon cause of secondary open angle glaucoma and was first described by Minas and Podos, ${ }^{1}$ although this entity is now termed Radius-Maumenee syndrome. ${ }^{2,3,4}$ The regulation of intraocular pressure (IOP) is a function of: production of aqueous humour, resistance to aqueous outflow at the anterior chamber angles, and episcleral venous pressure. When the episcleral veins are dilated, the pressure in these veins becomes elevated. Prolonged elevation of episcleral venous pressure often causes reduction in aqueous outflow facility and this consequently causes secondary glaucoma. ${ }^{2,5}$ Episcleral venous pressure is relatively stable, except when an alteration in body position and certain diseases of the orbit, head, neck and mediasternum obstruct venous return to the heart or shunt blood from the arterial to the venous system. The precise interrelationship between episcleral venous pressure and aqueous humour dynamics is complex and not well understood. There are a variety of clinical conditions which cause the elevation of episcleral venous pressure, and these include those associated with: venous obstructions (examples of these are dysthyroid ophthalmopathy, ${ }^{7}$ anterior and posterior scleritis, tenonitis, superior vena cava syndrome and those obstructing the orbital venous drainage like retrobulbar tumour and cavernous sinus thrombosis ${ }^{5,8}$ ), arteriovenous fistulas (from carotid-cavernous fistulas, ${ }^{9}$ orbital varices ${ }^{10}$ and Sturge Weber's syndrome ${ }^{11}$ ), and idiopathic elevated episcleral venous pressure, if the cause of the dilated episcleral vein cannot be found. ${ }^{2,3,5,8}$ IDEV is said to be due to a congenital abnormality in vasculature ${ }^{3}$ or localized venous obstruction in the region of the extraocular muscles. ${ }^{12}$ It can occur as a unilateral or a bilateral condition ${ }^{8}$ and at any age, although all the cases reported have been in persons over 20 years of age. There is no known sex predilection, but the majority of cases reported were among women. ${ }^{8}$ There is familial predisposition in IDEV, and it has been described in a mother and her daughter.

Idiopathic dilated episcleral vein (IDEV) is rare and is an uncommon cause of secondary glaucoma. To the best of our knowledge this is the first case reported in Nigeria.

\section{CASE REPORT}

A 69-year-old woman presented in OOUTH eye clinic four years ago with a one-year history of blurring of vision, pain, redness and a growth in her right eye. She was found to have dilated episcleral veins in her left eye. There was no antecedent history of trauma to the orbit, head or neck, no history of proptosis - either persistent or intermittent, no history of anterior neck swelling or symptoms suggestive of dysthyroid orbitopathy, and no family history of such eye disease. She was a controlled hypertensive but not diabetic.

${ }^{*}$ Correspondence: TO Otulana, Department of Ophthalmology, Faculty of Clinical Sciences, Obafemi Awolowo College of Health Sciences, 
On examination, her presenting visual acuities were 6/9 in each eye. She had a right nasal vascular pterygium. The left conjunctiva was hyperaemic with dilated and tortuous episcleral vessels. There was no obvious eye lid, periorbital or facial oedema or cyanosis. There were no dilated veins on the head, neck, chest or upper extremities and no demonstrable proptosis. Vasalva manoeuvre was negative. No conjunctival chemosis or episcleral haemangioma was seen, no bruit, no ocular motor nerve palsy and no demonstrable orbital mass. There was no facial lesion suggestive of Sturge Weber's syndrome. The corneae were clear with normal anterior chamber depths, pupils reacted briskly to light. She had early lens opacities. The optic discs were pink with cup-disc ratio (CDR) of 0.2 right, 0.3 left. Her intraocular pressures were $25 \mathrm{mmHg}, 50 \mathrm{mmHg}$ right and left eye respectively. At this time, she had no glaucomatous optic nerve damage or visual field defect on the Fieldman visual field analyzer. Gonioscopy revealed bilateral open angles but with blood in the left canal of Schlemm. Her left intraocular pressure was not controlled on tab acetazolamide $250 \mathrm{mg}$ thrice daily and drops of $0.5 \%$ timolol maleate twice daily. She had a left uneventful trabeculectomy ten months after commencement of medical treatment. In spite of a functioning bleb, her intraocular pressure fluctuated between 26 and $28 \mathrm{mmHg}$. Her right intraocular pressure dropped to between 12 and $16 \mathrm{mmHg}$ on medical treatment. She was recommenced to use ocular hypotensive agents, timolol $0.5 \%$ and the maximum dose of acetazolamide in her left eye. Three years after, she developed non specific visual field changes in her left eye and her CDR had increased from 0.3 to 0.5 , while the right still remained 0.2. Her vision at this time was $6 / 12$ in each eye due to increasing cataract. She was reviewed by the neurologist and no obvious neurologic deficit was found. Special radiological investigations, e.g. computerized tomography, magnetic resonance imaging and angiographic studies were not done because of financial constraint. Figures 1 and 2 show the left eye of the patient four years post trabeculectomy. The eye was chronically red with dilated and tortuous episcleral vessels.

\section{DISCUSSION}

Patients with IDEV may present to the ophthalmologist with chronic redness of the eye, without discomfort, discharge or allergic symptoms, ${ }^{13,}{ }^{14}$ visual impairment, or visual loss from secondary glaucoma. Conversely, it can be an incidental finding as evident in this patient who came into the clinic because of a vascular pterygium in the fellow eye. IDEV is a diagnosis of exclusion, ${ }^{6}$ and the possibility of carotid-cavernous fistula, orbital varix or other retro-ocular tumour had been excluded in this patient from the history and examination.

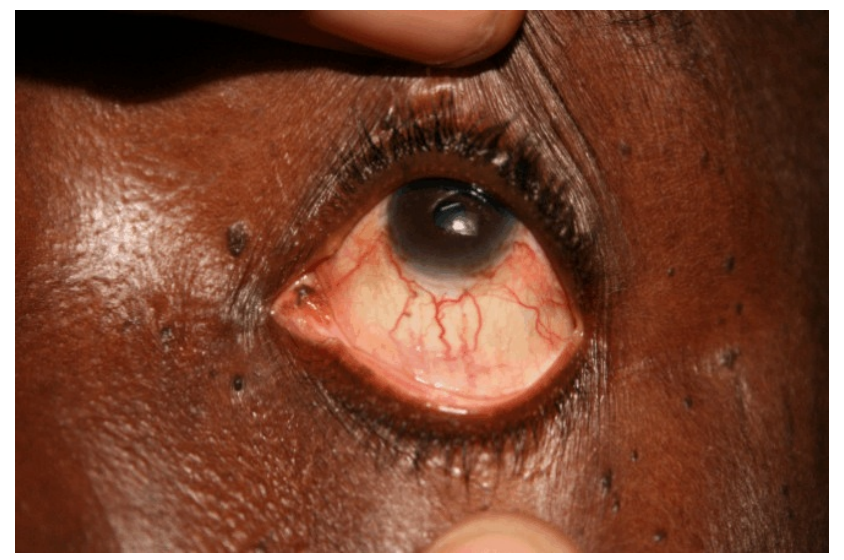

Figure 1. Left eye in up gaze. Note dilated episcleral vessels and characteristic redness.

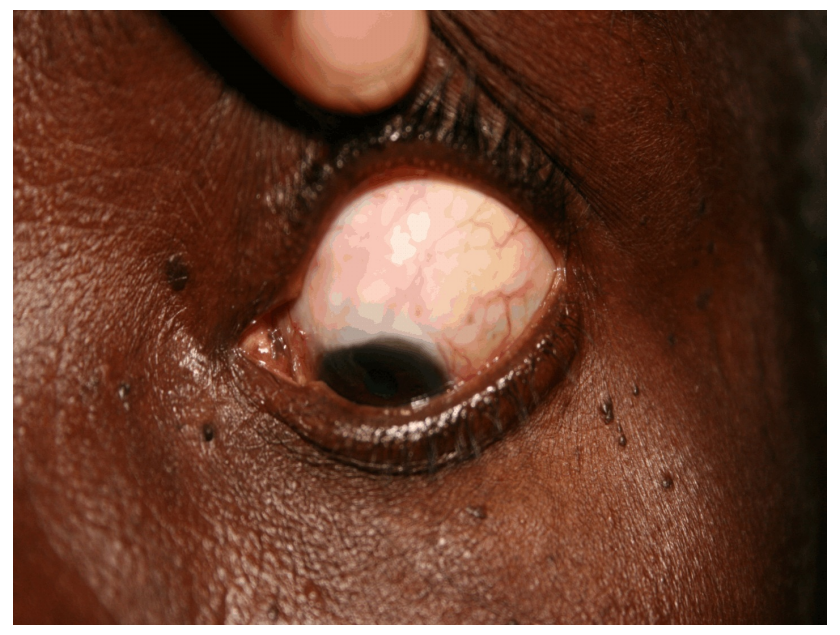

Figure 2. Left eye in down gaze. Four years post trabeculectomy Note the bleb, dilated episcleral vessels and characteristic redness.

The anterior segment in this patient appeared normal except for the vascular abnormality of the episcleral vessels in the left eye. The anterior chamber angle was open but with blood in the Schlemm's canal of the affected eye as found by other researchers. ${ }^{3,15}$ This may be a non-specific finding because it can be seen in $20 \%$ of normal eyes, but blood in the Schlemm canal was reported to be associated with episodic elevation of IOP. ${ }^{16}$ The facility of aqueous outflow may be normal in patients with IDEV, but this was not measured in this patient because there was no equipment to do so.

Catheter arteriography, neuroimaging such as computerized topographic scan and with enhancement, magnetic resonance imaging, other angiographic studies like selective carotid angiography, orbital venography, 
flourescien angiography to exclude lesions of the vortex veins are all useful investigations to determine the aetiology of dilated episcleral vessels. Orbital ocular Doppler imaging is important in imaging orbital and ocular blood flow, especially in cases of fistula and varix. ${ }^{3,13,15,17}$

Simple slit lamp examinations, tonographic measurement, visual field testing and orbital ultrasonography are also useful in the evaluation of these patients. $^{15}$ In our environment, where equipment for investigation is unavailable, inaccessible or unaffordable, a good clinical judgment based on a detailed history and a thorough physical examination will enable practitioners to makea reliable diagnosis.

Treatment of glaucoma in IDEV has focused on drugs which lower IOP by aqueous suppression while drugs that improve outflow are rarely effective. ${ }^{3}$ Surgery, in the form of trabeculectomy, sclerectomy or microsurgical sinusotomy, has also been employed. ${ }^{18,19,20,21}$ It is important to note that even when the IOP is controlled by surgery, dilation and tortuosity of episcleral veins may or may not resolve $e^{9,22}$ as was seen in our patient.

\section{CONCLUSION}

In the case reviewed in this study, all the typical features of dilated and tortuous episcleral veins were present but no cause could be found. Secondary glaucoma which ensued required further medical treatment as trabeculectomy alone appeared ineffective. A diagnosis of Radius-Maumenee Syndrome should be considered in cases of chronically red eye without symptoms of allergy, discomfort or discharge, especially when there is asymmetrical or unilateral elevation of intraocular pressure.

\section{REFERENCES}

1. Minas TF, Podos SM. Familial glaucoma associated with elevated episcleral venous pressure. Arch Ophthalmol 1968; 80: 202-8.

2. Groh MJ, Kuchie M. Idiopathic episcleral venous stasis with secondary open angle glaucoma (Radius-Maumenee Syndrome). Klin Monatsbi 1997; 211: 131-2.

3. Foroozan R, Buono LM, Savino PJ, Sergott. Idiopathic dilated episcleral veins and increased intraocular pressure. British Journal of Ophthalmology 2003; 87: 652-4.

4. Radius RL, Maumenee AE. Dilated episcleral vessels and open angle glaucoma. Am J Ophthalmol 1978; 86: 31-5.

5. Jorgensen JS, Guthoff R. The role of episcleral venous pressure in the development of secondary glaucoma. Klin Monatsbi Augenheilkd 1988; 193: 471-5.
6. Bigger JF. Glaucoma with elevated episcleral venous pressure. South Med J 1975; 68: 1444-8.

7. Goldberg I. Thyroid eye disease and glaucoma. Journal of Glaucoma 2003; 12: 494-6.

8. De Keizer RJW. Secondary vascular glaucoma. Documenta Ophthalmologica 1983; 56: 195-202.

9. Kiyotaka Ishijima, Kenji Kashiwagi, Kenichi Nakano, Takashi Shibuya, Toyoaki Tsumura, Shigeo Tsukahara. Ocular manifestation and prognosis of secondary glaucoma in patients with carotid-cavernous fistula. Japanese Journal of Ophthalmology 2003; 47: 603-8.

10. Kollarits CR, Gaasterland D, Dichiro G, Christiansen J, Yee RD. Management of a patient with orbital varices, visual loss, and ipsilateral glaucoma. Ophthal Surg 1977; 8: 54-62.

11. Phelps CD. The pathogenesis of glaucoma in Sturge Weber's syndrome. Ophthalmology 1978; 85: 276-86.

12. Jorgensen JS, Guthoff R. Pathogenesis of unilateral dilated episcleral vessels and increase in intraocular pressure. Klin Monatsbi Augenheilkd 1987; 190: 428-312.

13. Tolusan ED, Fishbein SL, Schwartz B. Increased pressure of dilated episcleral veins with open angle glaucoma without exophthalmos. Ophthalmology 1983; 90: 257-65.

14. Keltner JL, Gittinger JW Jr, Miller NR. et al. A red eye and high intraocular pressure. Surv Ophthalmol 1987; 31: 328-36.

15. Lanzl IM, Welge-Luissen U, Spaeth GL. Unilateral openangle glaucoma secondary to idiopathic dilated episcleral veins. Am J Ophthalmol 1996; 121: 587- 9.

16. Horowitz ER, Forbes M, Podos MS, Tsai JC. Episodic elevation of intraocular pressure associated with blood in the Schlemm's canal. Arch Ophthalmol 2004; 122: 1230-2.

17. Lieb WE, Merton DA, Shields JA, Cohen D, Goldberg BB. Colour Doppler imaging in the demonstration of an orbital varix. British Journal of Ophthalmology 1990; 74: 305-8.

18. Peter E. Libre. Nonpenetrating filteration surgery and goniopuncture (Staged trabeculectomy) for episcleral venous pressure glaucoma. Am JOphthalmol 2003; 136: 1172-4.

19. Guven D, Karkurt A, Ziraman I, Hasiripi H. Non penetrating deep sclerectomy in unilateral open-angle glaucoma secondary to idiopathic dilated episcleral veins. Eur J Ophthalmol 2002; 12: 66-8.

20. Bellows RA, Chylack LT, Epstein DL, Hutchinson BT. Choroidal effusion during glaucoma surgery in patient with prominent episcleral vessels. Arch Ophthal 1979; 97: 493- 7.

21. Bhagat N, Lim JI, Minckler DS, Green RL. Posterior uveal effusion syndrome after trabeculectomy in an eye with ocular venous congestion. Br J Ophthalmol 2004; 88: 153-4.

22. Grishaber MC, Dubler B, Knodel C, Killer HE, Flammer J, Orgul S. Retrobulbar blood flow in idiopathic dilated episcleral veins and glaucoma. Klin Monatsbi Augenheilkd 2007; 224: 320-3. 